\title{
The Future of Co-crystallisation: A New Workflow Based on Al Predictions, the Crystal16 Platform and Electron Diffraction
}

\author{
C. Guguta ${ }^{1}$, D.D.P.W. Stam ${ }^{1,2}$, A. E. Lanza ${ }^{2}$, G. Santiso-Quinones ${ }^{2}$, G. Steinfeld², D. Ungur ${ }^{3}$, C. lordache $^{3}$, \\ M. Pop ${ }^{3}$
}

${ }^{1}$ Technobis Crystallization Systems BV, Pyrietstraat 2, 1812 SC Alkmaar, The Netherlands, ${ }^{2}$ ELDICO Scientific AG, 5234 Villigen Switzerland

${ }^{3}$ TeraCrystal, Donat, no. 67 - 103, Cluj Napoca, Romania

carmen.guguta@technobis.com

Febuxostat (FB) is a poorly water-soluble BCS class II drug that is used for the treatment of the inflammatory disease arthritis urica (gout). FB has a rich solid form landscape, including many polymorphs, solvates, salts and a few co-crystals [1,2]. With the aim of improving the aqueous solubility of FB we expanded the search for novel salts and co-crystals by applying modeling techniques followed by directed crystallization experiments. Novel salt and co-crystal forms of FB were obtained in a controlled manner using the Crystal16 platform [3]. Making use of the integrated transmission technology together with 16 parallel reactors at a volume of $1 \mathrm{~mL}$, the Crystal16 easily allows the scientist to assess salt or co-crystal formation.

The salt/co-crystal formation was evidenced by powder X-ray diffraction and differential scanning calorimetry. Aqueous powder dissolution was carried out to determine if solubility improvement is achieved. Within the scope of this workflow, the nanocrystalline powders were not ideal for crystal structure elucidation from powder/single crystal X-ray diffraction but suited for electron diffraction experiments [4 -5 ]. By using a dedicated electron diffractometer [6], the crystalline structures of these materials were easily accessible.

Here we report on the successful crystallization and characterization of pharmaceutical relevant co-crystals using a new workflow: AI (artificial intelligence) predictions [7], the Crystal16 platform and an electron diffractometer.

[1] Maddileti D., Jayabun S. K., Nangia A. (2013) Crystal Growth \& Design 13 (7), 3188.

[2] Li L. Y., Du R. K.,. Du Y. L, Zhang C. J., Guan S., Dong C. Z., Zhang L. (2018) Crystals 8 (2), 85.

[3] Li W., de Groen M., Kramer H. J. M., de Gelder R., Tinnemans P., Meekes H., and ter Horst J. H. (2021) Cryst. Growth Des. 21 (1), 112.

[4] Andrusenko I., Potticary J., Hall S. R., Gemmi M. (2020) Acta Cryst. B76, 1036.

[5] Hamilton V., Andrusenko I., Potticiary J., Hall C., Stenner R., Mugnaioli E., Lanza A. E., Gemmi M., Hall S. R.(2020) Cryst. Growth Des. 20, 4731.

[6] ELDICO Scientific AG has developed a dedicated device for electron diffraction experiments. This device, its capabilities, and advantages over (modified)-TEMs will be showcased in this congress too. A scientific publication on a dedicated device for ED experiments is in preparation too.

[7] Devogelaer J.J, Meekes H., Tinnemans P., Vlieg E., de Gelder R. (2020) Angew.Chem. Int.Ed. 59, 21711.

Keywords: co-crystal, electron diffraction, artificial intelligence, crystallisation, solubility 